\title{
QUALITY OF LIFE AND MALADJUSTMENT ASSOCIATED WITH HAIR LOSS IN WOMEN WITH ALOPECIA ANDROGENETICA
}

\author{
J. Van der Donk, ${ }^{1}$ J. A. M. Hunfeld, ${ }^{2}$ J. Passchier, ${ }^{1}$ K. J. Knegt-JunK ${ }^{3}$ and C. Nieboer ${ }^{4}$ \\ 'Department of Medical Psychology and Psychotherapy, Erasmus University, Rotterdam, ${ }^{2}$ Department \\ of Gynaecology, University Hospital, Rotterdam-Dijkzigt, ${ }^{3}$ Department of Dermatology and Venereology. \\ University Hospital, Rotterdam-Dijkzigt and 'Department of Dermatology, Free University, Amsterdam, \\ The Netherlands
}

\begin{abstract}
Quality of life and maladjustment related to hair loss were studied by means of a standardized interview in a group of 58 women with alopecia androgenetica who applied for treatment at the Department of Dermatology. The hair loss was found to have a negative influence on the quality of life on the majority of them. In $88 \%$, hair loss had negative effects on their daily life; in about $75 \%$, the hair problems were manifested in negative self-esteem and about $50 \%$ experienced social problems. General psychosocial maladjustment in relation to hair loss was indicated in almost one-third of the women.
\end{abstract}

Key words -alopecia androgenetica, maladjustment, quality of life

\section{INTRODUCTION}

Compared to the gradually increasing number of studies being published on men with alopecia androgenetica [1-3], the lack of attention being paid to women with this affliction is remarkable.

More psychosocial problems can be expected in women than in men because a smaller proportion of women are affected, which emphasizes their deviation from the norm. Venning and Dawber [4] found that $13 \%$ out of 564 premenopausal women showed hair loss on the scalp, while in men this percentage was about $45 \%$ [5].

A second reason for expecting more psychosocial problems in women with alopecia androgenetica than in men is that our cultural norms emphasize the relevance of physical attractiveness for women in particular.

So far, the psychological and social problems of women with alopecia androgenetica have mainly been described anecdotally [6]. Eckert [7] studied women with diffuse alopecia and found that $20 \%$ of them were suffering from severe marital problems or were overtly depressed. van der Donk et al. [8] compared women with alopecia androgenetica to women with a non-visible dermatological disorder and to male subjects with alopecia androgenetica, using standardized psychological instruments. They found elevated scores for self-satisfaction and social inadequacy in comparison with the women with non-visible dermatological complaints. In addition, the women with alopecia androgenetica scored higher on inadequacy, rigidity, injuredness and general psychological maladjustment and lower on selfevaluation and self-esteem than the men with alopecia androgenetica. Finally, they showed more psychosocial problems, which they attributed to their hair loss, than any of the other groups.

The present study is a continuation of the previous one by van der Donk et al. [8] and explores the character and extent of psychosocial problems related to alopecia androgenetica in the aforementioned 58 women with alopecia androgenetica more deeply, in order to assess the influence of hair loss on their quality of life.

As the sensitivity of standardized generic quality of life instruments, such as the Nottingham Health Profile, is highest mainly in the severe range [9], data were collected via in-depth interviews.

The use of an interview method also gives detailed information on the situations in which the problems are experienced, which can be used for behavioural management.

As this study was concerned solely with problems which can be attributed to alopecia androgenetica, no control group was included.

Our study questions were

(1) What problems exist in various life areas in the women with alopecia androgenetica who applied for treatment?

(2) How many women with alopecia androgenetica show general psychosocial maladjustment which is attributable to alopecia androgenetica?

\section{METHOD}

\section{Subjects}

Subjects who applied for treatment for their hair loss at the Department of Dermatology at the University Hospital Dijkzigt in Rotterdam and the Free University Hospital in Amsterdam were invited 
by letter to participate in a double-blind clinical trial. The trial had the purpose of investigating the effect of $2 \%$ minoxidil lotion treatment and will be published elsewhere. From the respondents, 70 healthy, dark-haired women, aged between 18-45 years and with a minimum duration of hair loss of 6 months, were selected by dermatologists (K.J.K.J, C.N), on the basis of the following criteria for female pattern alopecia:

(1) diffuse thinning in the fronto-parietal area of the scalp, both with or without temporal recession,

(2) grades I and II according the Ludwig scale [10].

Subjects were assigned at random, in a doubleblind fashion, to either a $2 \%$ minoxidil lotion treatment group or to a placebo treatment group. Of the 70 women who met these criteria, ${ }^{*} 58$ agreed to participate in our psychological study, resulting in a response rate of $83 \%$. The protocol of the study was reviewed and accepted by the Medical Ethics Committee of the University Hospital RotterdamDijkzigt. The subjects received a small reward for their invested effort (cquivalent of U.S. \$5).

\section{Procedure}

The data were collected using an interview with questions related to the significance of hair, the problems resulting from hair loss and psychosocial maladjustment. The interview was held in the 9 th month of the medical study and had a retrospective character, i.e. the questions referred to the situation 1 month before the start of treatment. It took about 30 min and was carried out at the Department of Dermatology by a trained female psychologist (J.A.M.H.) in view of the possibility of specific female problems.

\section{Instruments}

The interview consisted of two parts and was semi-structured. It was based on the experience gained during the explorative interviews with subjects

*For the clinical trial, the applicants also had to have: a normal menstrual history for their age and show no abnormalities on the following tests: physical examination (including normal pelvic examination and cervical cytology conducted within 6 months prior to the start of the treatment), gynaecological, electrocardiogram, echocardiogram. chest X-ray and laboratory evaluations (haematology, serum biochemistry and urine analysis). Subjects with hypertension (>140/90 mm Hg); scalp disease; abnormal ovarian function and/or evidence of ovarian enlargement; a history of cardiac, renal, hepatic, or endocrine disease; and those using cytotoxic agents, corticosteroids, antihypertensive, peripheral vasodilatory, bronchodilatory, antiepileptic medications and hair restorers, were excluded from the medical part of the study. Also excluded were applicants with clear-cut psychiatric symptoms, those who were unable to follow or understand the study instructions, those with light coloured, advanced gray, dyed and/or extremely curly hair. with alopecia androgenetica in the pilot phase of the study. The first part consisted of open questions on the main life areas so that the subject could bring forward topics which were related to hair loss. A written report on this part of the interview was made afterwards for later analysis. The second part of the interview consisted of a number of closed questions, in particular about the subject's behaviour and feelings in specific situations. These situations were adopted from the pilot phase of the study and were only presented to the subject if they had not already been discussed in the first part of the interview.

\section{Data analyses}

To answer the first research question on the effect on quality of life, the answers were analysed descriptively by frequency counts of the psychological problems. For the second research question on the general psychosocial maladjustment of the women, the written records were analysed to review the women's adjustment to their hair loss. Two independent judges (J. vd D., J.P.) classified the adjustment into (1) adaptive and (2) maladaptive adjustment. They used the following operationalizations:

(1) Good adjustment to hair loss was expressed by the absence of any general effect of the hair loss on the subject's feelings and her participation in daily activities. A number of specific negative aspects related to hair loss could be present. In addition, the adjustment led to positive expectations of the subject about the future.

(2) Maladjustment to hair loss was expressed by preoccupation with the negative aspects of hair loss, leading to manifold unpleasant and troubled feelings and to the avoidance of normal daily activities. Furthermore, it led to negative expectations about the future.

On the basis of this classification, the subjects were divided into three categories by the judges: (A) predominantly well-adjusted subjects, (B) predominantly maladjusted subjects and (C) subjects who showed both well-adjustment and maladjustment without an overall predominance of either one.

The agreement between the judges on the classification of the women according to their adjustment was $86 \%$ initially. After sharpening of the criteria, the remaining $14 \%$ were discussed again resulting in an agreement of $94 \%$. There was no concordance in $6 \%$.

\section{RESULTS}

\section{Subject characteristics}

The characteristics of the subjects are shown in Table 1.

\section{Quality of life}

Table 2 shows the frequency and percentage of psychosocial problems mentioned during the interview and categorized by life area. In the following 
Table 1. Characteristics of women with alopecia androgenetica

\begin{tabular}{lrrrr}
\hline Characteristics & $M$ & (SD) & $N$ & $\%$ \\
\hline Age (in years) & 36 & $(6.6)$ & & \\
$\quad$ History of hair loss (in years) & 12 & $(7.5)$ & & \\
History of visible hair loss (in years) & 9 & $(7.0)$ & & \\
Marital status* & & & & \\
With a partner & & 36 & 63 \\
Single & & 12 & 21 \\
Divorced & & 8 & 14 \\
Widow & & 1 & 2 \\
Educational level & & & \\
Low (secondary education or less) & & 15 & 26 \\
High (more than secondary education & & 43 & 74 \\
\hline * Characteristics of one subject are missing. & & &
\end{tabular}

paragraphs, the content of the table is clarified by presenting typical statements made by the subjects in each life area.

\section{The attitude to hair and hair loss}

More than $62 \%$ of the subjects felt that hair is an important aspect of physical appearance and/or an important means of self-expression. This was illustrated by statements such as: "If you are employed as some sort of representative, you can't go round with wispy, thin hair or bald spots." "My hair loss forces me to wear a timid hair-style, but I feel much wilder inside." "A wife without hair is no real wife for a man." "If I had beautiful hair, it might give me more self-confidence and then I wouldn't need to be so uncivil to everyone". For $15 \%$ of the women, hair loss formed a physical handicap and they wished to be released from the wig or prothesis which they regarded as an artificial part of their body.

Table 2. Psychosocial problems associated with hair loss: number of responses and percentage for each life area and response category

\begin{tabular}{lcc}
\hline & $\begin{array}{c}\text { Absolute } \\
\text { number }\end{array}$ & \\
Life area and response category* & of responses & $\%$ \\
\hline Attitude to hair and hair loss & 54 & 93 \\
Hair forms an expression of oneself & 36 & 62 \\
Major factor in female appearance & 22 & 41 \\
Hair loss is a handicap & 9 & 15 \\
Negative effects on self-esteem & 42 & 72 \\
Less attractive & 33 & 57 \\
Reduced confidence & 31 & 54 \\
Uncertainty & 19 & 33 \\
Shame & 15 & 26 \\
Inferiority complex & 12 & 21 \\
Feeling old & 7 & 12 \\
Negative activities in daily life & 51 & 88 \\
Conceals hair loss & 40 & 69 \\
Takes more care & 35 & 60 \\
Pays more attention to the hair of others & 17 & 29 \\
Takes care where to sit & 13 & 22 \\
Goes out less or stays at home & 10 & 17 \\
Negative feelings during social contact & 28 & 48 \\
Is worried & 15 & 26 \\
Is afraid of remarks about hair loss & 9 & 16 \\
Inhibited & 7 & 13 \\
Inferiority complex & 4 & 7 \\
Is jealous of others & 4 & 7 \\
\hline
\end{tabular}

"Subjects could give more than one answer.

$\uparrow$ Responses given by fewer than $5 \%$ of the subjects are not mentioned in this table.

\section{Negative effects on self-esteem}

In most of the women ( $72 \%)$, the hair loss affected their self-esteem in a negative way. This was expressed in terms of decreased self-confidence, in uncertainty, shame, feelings of inferiority and feeling old. Over half $(57 \%)$ felt less attractive as a result of the hair loss.

\section{Negative activities in daily life}

The hair loss affected the behaviour of a large proportion of the women $(88 \%)$. They felt restricted and adopted a variety of precautionary measures in relation to their hair loss. Several subjects said that they were tense in shops and other public places and, for instance, suffered from neck cramp from holding up their head to prevent others from discovering the hair loss. One subject told us that she always pays cash so that the cashier cannot see her head while she writes a cheque. Most women preferred not to go swimming and stayed at home when it was raining or very windy. Walking in the sun appeared to be at least equally as distressing, "because the sun shines through your hair and burns your head". But indoors, the hair loss could be troublesome as well: "If I notice that my hair is untidy, I avoid getting involved in a dispute".

Many women tried to hide their hair loss $(69 \%)$ by frequent washing, drying, or cutting and sometimes they rubbed the scalp with powder in order to make it less discernible from the hair. One woman said, "I wear a headscarf to hide my hair loss and not for a religious reason." Nearly one-third of the women stated that they frequently looked at other women's hair and $17 \%$ said that they avoided going out, especially when they had started to lose their hair. Twenty-two per cent of the women avoided particular locations, such as places with strong illumination, the centre of a terrace where the sun shines brightly, or the centre of a room where other people might see the top of their head.

\section{Negative feelings during social contact}

For $48 \%$ of the subjects, the hair loss formed a problem socially. More than a quarter felt worried that others would see the thinning patches: "I always feel nervous in company because I feel like I have a large, round bald spot on my head." One out of every seven women was afraid that others would make remarks about the hair loss: "I keep quiet particularly at work, because the moment they make a remark about my hair, I feel very small." The women felt offended by jokes or remarks about their hair loss and found them very improper, such as: "Hey, old woman" or "I can see your scalp!" For most of the women it was important who made these remarks. A number of them used to crack cynical jokes about themselves, such as: "Any punk would be jealous. I hardly need to do anything to get this hairstyle." 


\section{Adjustment}

The written reports showed that $36 \%$ of the subjects were predominantly well-adjusted regarding coping with their hair loss. This approach was illustrated by one of the women in the following words:

In company I am usually more worried about being in a bad mood than about my hair loss. Of course, suddenly having thoughts about the hair loss can soon change that. As such, the hair loss isn't that much of a problem to me. It doesn't automatically mean that I would start thinking about a wig if I was to go bald-I regard my hair as a means of distinguishing myself from others. Neither is it my intention to use some sort of medication for the rest of my life against hair loss. I prefer to spend my money on other things.

The reports also showed that $29 \%$ of the women were predominantly maladjusted regarding their hair loss. One of them told us, for example:

In company I always feel that other people are looking at me all the time and I hate that. That is why I prefer to stay at home. I've never been what you might call attractive, but now with my hair like this, it's even worse. A woman without hair isn't really a woman anymore. And if I wasn't already aware of that, the other people around me would soon let me know! So, I am afraid that I will go completely bald. I think that if my hair doesn't stop falling out, I will go down hill very fast psychologically.

Both well-adjustment and maladjustment were observed in $29 \%$ of the women. The general trend in these cases was, for instance:

I always feel worried in company and I am afraid that my wig won't stay straight. But when I get chatting, the feeling disappears. The looks other people give me make me terribly annoyed and as soon as they start talking about their own hair-styles, I run for cover! I have always felt unattractive and fat even as a child and when I started to lose my hair too, I wouldn't go out to play anymore. I locked myself in and began to eat. But nowadays I wear a wig and have the courage to go out again. Perhaps I will try hair fusion some time. A new wig really boosts my self-confidence. Luckily, I am a cheerful person.

\section{DISCUSSION}

Scalp hair plays a major role in determining physical attractiveness and is an important aspect in interpersonal contact, as it is the first and most directly visible source of non-verbal information $[11,12]$. Scientific studies have demonstrated that individuals who are rated as being physically attractive are thought to be more successful [13], to enjoy more respect [14], to have more friends, to be more happily married [15] and to be socially better adjusted [16] than less physically attractive individuals. Physical attractiveness also seems to have intrapersonal consequences, as indicated by the positive correlation with self-esteem and the negative correlation with neuroticism [17].

Studies on individuals who think themselves physically unattractive were almost exclusively conducted on clinical populations. van Keep [18] reported that the majority of the subjects in his study with a visible dermatological disorder avoided social contact and lost touch with acquaintances and friends. Baardman [19] found that nearly $20 \%$ of the clients at a clinic for neurosis were negatively preoccupied by their appearance, which caused social avoidance behaviour, poor physical hygiene, etc. In brief, feelings of physical unattractiveness seem to be related to many negative psychosocial aspects. These negative aspects were also found in this study on women who applied for treatment for alopecia androgenetica.

A large number of the women in this study experienced a negative effect on their quality of life as a consequence of this affliction. These problems manifested themselves in the effort taken to hide the hair loss, in their taking care where to sit and in avoiding company and activities, in inhibited behaviour, feelings of envy, lost self-confidence and feelings of insecurity in company. Most of them had sought help for their alopecia androgenetica: the majority had consulted their general practitioner or a dermatologist. In general, they considered the care offered to be insufficient. For this reason, many of the women felt powerless and referred to their worries about the cause and possible progressive nature of the hair loss as most unpleasant. A possible case in point was that three women in our study group considered their hair loss to be comparable with a physical impairment, such as the loss of a limb.

The psychosocial problems of the women with alopecia androgenetica in this study appeared to be comparable with the psychological problems of individuals with a (visible) dermatological disorder, such as acne, eczema and psoriasis. The social life of $40 \%$ of the individuals with one of these dermatological disorders was affected by the disorder [20], whereas in our group this percentage was $50 \%$.

\section{Adjustment}

It was striking that $29 \%$ of the women in our study group were unable to cope with their alopecia androgenetica and experienced severe maladjustment. Furthermore, $36 \%$ of the women were predominantely wcll-adjusted and $29 \%$ showed both well-adjustment and maladjustment. While general practitioners in The Netherlands reported that $50 \%$ of the patients who presented with alopecia androgenetica had psychological problems, they nevertheless used the same policies for patients with and without psychological problems [21]. The authors of this study conclude that many patients with alopecia androgenetica do not receive sufficient psychological support from their general practitioner in a psychological respect. As patients with alopecia androgenetica primarily seek help and comfort in the medical field, psychological support, such as information, emphatic listening and behavioural advice regarding the above problems, ought to be supplied by the general practitioner and the dermatologist. 
The question arises as to whether the problems and adjustment of the women with alopecia androgenetica in this study can be extrapolated to the general population of women suffering from this affliction. A further epidemiological study on a nonclinical group of women with alopecia androgenetica might help to find the answer to this question.

Acknowledgements - This study was supported by a grant from The Upjohn Company, Kalamazoo, MI. Mrs Goudsmit provided the administrative support.

\section{REFERENCES}

1. Passchier J., Donk van der J., Dutree-Meulenberg R. O. G. M., Stolz E. and Verhage F. Psychological characteristics of men with alopecia androgenetica and effects of treatment with topical minoxidil: an exploratory study. Int. J. Dermat. 27 (Suppl), 441-446, 1988.

2. Donk van der J., Passchier J., Dutree-Meulenberg R. O. G. M., Stolz E. and Verhage F. Psychologic characteristics of men with alopecia androgenetica and their modifation. Int. J. Dermat. 30, 22-28, 1991.

3. Gosselin C. Hair loss, personality and attitudes. Pers. individ. Diff. 5, 365-369, 1984.

4. Venning V. A. and Dawber R. P. R. Patterned androgenetic alopecia in women. J. Am. Acad. Dermat. 18 1073-1077, 1988.

5. Hamilton J. B. Patterned loss of hair in man: types and incidence. Ann. N.Y. Acad. Sci. 53, 708-728, 1951.

6. Beard H. O. Social and psychological implications of alopecia areata. J. Am. Acad. Dermat. 14, 697-700, 1986.

7. Eckert P. Diffuse hair loss in women: the psychopathology of those who complain. Acta psychiat. scand. 53, 321-327, 1976.

8. Donk van der J., Passchier J., Knegt-Junk C., WegenKeijser van der M. H., Nieboer C., Stolz E. and Verhage
F. Psychological characteristics of women with androgenetic alopecia: a controlled study. Br. J. Dermat. 125 , 248-252, 1991.

9. Hunt S. M., McKenna S. P. and McEwen J. The Nottingham Health Profile: subjective health status and medical consultations. Soc. Sci. Med. 15, 221-229, 1981.

10. Ludwig E. Classification of the types of androgenetic alopecia (common baldness) occurring in the female sex. Br. J. Dermat. 97, 247-254, 1977.

11. Patzer G. L. The Physical Attractiveness Phenomena. Plenium Press, New York, 1985.

12. Hatfield E. and Sprecher S. Mirror, Mirror: The Importance of Looks in Everyday Life. State University of New York Press, Albany, 1986.

13. Dion K. K., Berscheid E. and Walser E. What is beautiful is good. J. Pers. soc. Psychol. 24, 285-290, 1972

14. Berscheid E. and Walster E. Beauty and the best. Psychol. Today 5, 42-46, 1972.

15. Kirkpatrick C. and Cotton J. Physical attractiveness, age and marital adjustment. Am. sociol. Rev. 16, 81-86, 1951.

16. Lerner R. M. and Lerner J. V. Effects of age, sex and physical attractiveness on child-peer relations, academic performance, and elementary school adjustment. Dev. Psychol. 13, 585-590, 1977.

17. Mathes E. W. and Kahn A. Physical attractiveness, happiness, neuroticism and self-esteem. J. Psychol. 90, 27-30, 1975.

18. Keep van $\mathbf{P}$. A. Isolation: the influence of skin diseases on social relationships. Int. J. Dermat. 13, 227-231, 1974.

19. Baardman I. Ingebeelde Lelijkheid. Free University Press, Amsterdam, 1989.

20. Jowett $S$. and Ryan T. Skin disease and handicap: an analysis of the impact of skin conditions. Soc. Sci. Med. 20, 425-429, 1985 .

21. Koning de E. B. G., Passchier J. and Dekker F. W. Psychological problems with hair loss in general practice and the treatment policies of general practitioners. Psychol. Rep. 67, 775-778, 1990. 